\title{
LA EVALUACIÓN FORMATIVA EN LA ENSEÑANZA DE HISTORIA, GEOGRAFÍA Y CIENCIAS SOCIALES
}

\author{
Gerardo SÁNCHEZ SÁNCHEZ ${ }^{1}$ \\ Thalía ESPINOZA CÁCERES \\ Nicole GUTIÉRREZ NÚÑEZ \\ Oliver LETELIER CONTRERAS \\ Karenn MORA VERGARA \\ Romina MORAGA ORELLANA \\ Paulina MUÑOZ VEGA
}

\section{RESUMEN}

En nuestro sistema educativo la evaluación de los aprendizajes es una práctica centrada en resultados, y, por tanto, en su función social. Actualmente, el decreto 67/2018 plantea el desafío con una evaluación formativa que debe iniciar su implementación el año 2020. La investigación cualitativa basada en un estudio de casos múltiples, analiza cómo se implementa la evaluación formativa en la enseñanza primaria de Historia, Geografía y Ciencias Sociales, en colegios de la provincia de Talca. Los resultados confirman la presencia de una función pedagógica de la evaluación, condicionada por la estandarización del sistema educacional.

PALABRAS CLAVES: Aprendizaje, Prácticas pedagógicas, Evaluación formativa, Retroalimentación, Estandarización.

\section{ABSTRACT}

In our educational system, the evaluation of learning is a practice focused on results, therefore, on its social function. Currently, the Decree 67/2018 poses the challenge with a formative evaluation that must begin its implementation in 2020. This qualitative research based on a multiple case study seeks to analyze how the formative evaluation is implemented at the teaching of History, Geography and Social Sciences, at schools in the province of Talca. The results indicate a pedagogical practice with the presence of the pedagogical function of evaluation, conditioned by the standardization of the educational system.

\footnotetext{
${ }^{1}$ Los autores pertenecen a la Facultad de Ciencias de la Educación - Universidad Católica del Maule - Chile C.E.gerignsan@gmail.com
}

Fecha de recepción del artículo: Julio 2020

Fecha de evaluación: Noviembre 2020 
KEYWORDS: Learning, Pedagogical Practices, Formative Evaluation, Feedback, Standardization.

\section{RÉSUMÉ.}

Dans notre système éducatif, l'évaluation de l'apprentissage est une pratique centrée sur les résultats, et donc sur sa fonction sociale. Actuellement, le décret 67/2018 prévoit de relever le défi avec une évaluation formative qui doit commencer sa mise en œuvre d'ici 2020. La présente recherche qualitative basée sur une étude de cas multiples cherche à analyser comment mettre en œuvre l'évaluation formative dans l'histoire de l'histoire. , Géographie et sciences sociales, dans les collèges de la province de Talca. Les résultats indiquent une pratique pédagogique avec la présence d'une fonction d'évaluation pédagogique, conditionnée par la standardisation du système éducatif.

MOTS CLÉS: Apprentissage, Pratiques pédagogiques, Évaluation formative, Feedback, Standardisation.

\section{INTRODUCCIÓN}

El nuevo Sistema Nacional de Evaluación de Aprendizaje promueve potenciar el uso pedagógico de la evaluación, entendiéndola en términos formativos, vale decir, como un "conjunto de acciones realizadas para recoger evidencia de lo que los estudiantes están aprendiendo en relación a las metas de aprendizaje y así poder retroalimentarlos y/o modificar la enseñanza" (Agencia de Calidad de la Educación 2018:16). Este nuevo sistema se encuentra sustentado en el decreto $\mathrm{N}^{\circ} 67 / 2018$, que entra en vigencia en el año 2020, el cual establece y regula normas mínimas nacionales sobre evaluación, calificación y promoción de los alumnos a nivel nacional que cursen la modalidad tradicional en la enseñanza básica en establecimientos municipales, y que en lo específico compromete un desafío hacia la evaluación formativa. En este sentido, surge la interrogante ¿Cómo los docentes implementan la evaluación formativa en la enseñanza de Historia, Geografía y Ciencias Sociales?, además de resultar interesante preguntarse, ¿Qué cambios viene a introducir a la práctica evaluativa de los profesores esta nueva reglamentación?, ¿Cuáles son las brechas y/o desafíos a los que se enfrentará el decreto en cuestión?, entre otras muchas interrogantes.

La presente investigación como parte de una primera aproximación descriptiva, busca indagar en el proceso de implementación de la evaluación formativa en la enseñanza de Historia, Geografía y Ciencias Sociales en docentes de cuatro establecimientos educacionales de la provincia de Talca. Para ello, se opta por una perspectiva metodológica cualitativa que procura rescatar los sentidos y significados otorgados por los sujetos, a través del uso de observaciones de clases y de entrevista, en donde se exponen nociones a partir de la experiencia que posea cada uno de ellos.

\section{PLANTEAMIENTO DEL PROBLEMA}

En Chile, el sistema escolar se ha caracterizado por un proceso evaluativo orientado hacia la medición y a la certificación de los logros. Si bien, es un tema con pocas fuentes de 
información, Contreras sostiene que "las escasas investigaciones realizadas dan cuenta de prácticas fuertemente influidas por una racionalidad técnica, en que se significa más bien la evaluación como medición, en el mejor de los casos como congruencia de objetivos, y que se usa preferentemente para calificar o para cumplir con trámites administrativos" (2017: 123).

El sentido social de la evaluación, vinculada a la acreditación del cumplimiento de aprendizaje, es una práctica ya instalada en nuestro país y las diversas pruebas estandarizadas lo confirman, condicionando y presionando la actuación del profesorado. A partir de las reformas establecidas estos últimos años, que orientan al sistema educativo en función de la equidad y calidad, se han definido políticas con la finalidad de promover el desarrollo integral de las y los estudiantes, tal como se establece en el decreto $\mathrm{N}^{\circ} 67 / 2018$, señalando la evaluación, como parte intrínseca del proceso pedagógico, la cual podrá usarse formativa y sumativamente, otorgándole mayor relevancia a la primera modalidad, y de esta manera monitorear y acompañar el aprendizaje de los estudiantes. Una mirada a la evidencia internacional, reflejada en los informes de la Organización para la Cooperación y Desarrollo Económico (OCDE) en su publicación Synergies for better learning (2013) señala que,

uno de los desafíos de los sistemas educativos es encontrar un balance entre las evaluaciones sumativas y formativas a través del fortalecimiento de los procesos de evaluación que suceden en las escuelas. Frente a este desafío se recomienda crear marcos de evaluaciones comprensivos, que incluyan una variedad de enfoques y propósitos evaluativos (Agencia de Calidad de la Educación, 2018, p. 12).

El decreto $N^{\circ} 67 / 2018$, establecido por el Ministerio de Educación, se centra en las normas mínimas nacionales sobre evaluación, calificación y promoción, deroga los decretos exentos N511 del año 1997, Nº112 de 1999 y N83 de 2001. En él se establecen una serie de artículos que dan a entender los reajustes respecto a la evaluación, la que tiene como inicio de implementación el año 2020, y pretenden fortalecer la función pedagógica de la evaluación, en términos de diversificar la evaluación y sus estrategias en orden a atender de mejor manera a la diversidad de los alumnos, gestionar la forma y los tiempos para la comunicación sobre el proceso, progreso y logros de aprendizaje a los alumnos, padres, madres, apoderados, favorecer instancias mínimas de comunicación, reflexión y toma de decisiones entre los diversos integrantes de la comunidad educativa centradas en el proceso, el progreso y los logros de aprendizaje de los alumnos.

El Ministerio de Educación por su parte, a través de sus Bases Curriculares y Programas de Asignatura, ofrece a los docentes orientaciones curriculares que en el ámbito de la evaluación comprometen la noción de proceso sistemático que involucra evaluación diagnóstica, formativa y sumativa.

La evaluación en el ámbito de la educación corresponde a un eje central del proceso de enseñanza y aprendizaje, ya que, permite evidenciar y monitorear el avance de los estudiantes con respecto a las metas de aprendizaje establecidas, y a la vez permite al docente reflexionar acerca de las prácticas y decisiones que toma en el desarrollo de cada objetivo.

Complementando lo anterior, la evaluación para Zabalza “es un proceso sistemático de conocimiento que implica como mínimo tres fases: Recogida de información. Valoración de la información recogida. Toma de decisión" (2007: 149).

Al centrarse en la evaluación desarrollada en el aula, esta se define como, 
una amplia gama de acciones lideradas por las y los docentes, como parte del proceso de enseñanza y aprendizaje, para que tanto ellas y ellos como las y los estudiantes puedan obtener evidencia sobre el aprendizaje e interpretarla para tomar decisiones que permitan promover el progreso de este y mejorar los procesos de enseñanza (Ministerio de Educación 2019: 28).

Por el contrario, al referirnos al concepto de evaluación, esta se comprende como un proceso que permite evidenciar el avance de las trayectorias de los estudiantes, con el objetivo de ajustar y re- direccionar la enseñanza si es necesario, es decir, se pretende avanzar hacia algo más que solo lo cuantitativo, dando una utilidad al resultado y tomar decisiones a partir de este. Para Contreras, la evaluación de los estudiantes constituye "un proceso de emisión de juicios acerca de la calidad de los aprendizajes, con el fin de, por una parte, certificar dichos aprendizajes (sentido sumativo) y, por otro, mejorar los procesos de enseñanza y aprendizaje (sentido formativo)" (2017: 120).

La manera en que se emplee la evaluación, ya se formativa o sumativamente, dependerá del objetivo que se pretende alcanzar. De este modo, el uso formativo o sumativo, se asocia con el sentido pedagógico y el sentido social respectivamente que posee la evaluación.

En la actualidad, se busca avanzar hacia el sentido pedagógico que tiene la evaluación, promoviendo de esta manera el desarrollo del aprendizaje. Cabe destacar, que un buen uso de la evaluación atiende a las características y a la diversidad que se presenta en el aula, y, por lo tanto, a los requerimientos del proceso de enseñanza y aprendizaje. En esta dirección, es que se pretende dar mayor uso formativo a la evaluación, ya que, como señala Calatayud una evaluación para el aprendizaje es aquella "orientada a pensar en la evaluación como un proceso que tiende a ser continuo y que destaca, sobre todo, el efecto retroalimentador para tratar de que el estudiante avance a partir de sus posibilidades e individualidades" (2019:170)

Considerando que toda evaluación supone un proceso de toma de decisiones, es lícito preguntar si la evaluación está permitiendo al profesorado reflexionar en torno a su práctica pedagógica con la intención de mejorar, y a los estudiantes, poder avanzar en autonomía y autorregulación.

Actualmente, la evaluación pretende avanzar a un cambio de concepción, ya que, en lo que respecta a políticas públicas, el cambio de decreto N`511 del año 1997 al decreto Nº67 del año 2018, desafía a los docentes a la actualización de sus prácticas evaluativas. Ahora bien, para lograr una efectividad en lo anterior es fundamental que se reconceptualicen los tres roles de evaluación: diagnóstica, sumativa y formativa. En ese escenario, es clave preguntarse qué rol juega efectivamente en las aulas la evaluación formativa en la enseñanza de Historia, Geografía y Ciencias Sociales, cómo es conceptualizada, qué fines persigue, y qué efectividad logra. Cuestión que necesariamente tensiona la naturaleza y sentido de las prácticas pedagógicas.

Prácticas pedagógicas

Dentro del aula, las prácticas pedagógicas comprometen acciones y responsabilidades institucionales y sociales. Entendiendo estas como elemento de transformación social, han de ser comprendidas tanto en su dimensión de proceso como de resultado. Desde la perspectiva de Muñoz, Villagra y Sepúlveda 
La práctica docente es de carácter social, objetiva e intencional. En ella intervienen los significados, percepciones y acciones de las personas involucradas en el proceso educativo (alumnos, docentes, padres, autoridades, entre otros). También intervienen los aspectos político-institucionales, administrativos y normativos, que en virtud del proyecto educativo de cada centro delimitan el rol del profesor. Es decir, la práctica docente supone una diversa gama de relaciones entre personas (2016: 80).

El ejercicio de la tarea docente exige que los profesores reflexionen en torno a lo que sucede y tomen decisiones adecuadas. En ese sentido, Alliaud sostiene que "quienes enseñan interpretan lo que sucede (en lo inmediato o a lo largo de un tiempo) para, a partir de allí, resolver lo que es posible y deseable hacer en determinadas circunstancias. Hoy más que nunca los docentes tienen que poder "leer", en el sentido de interpretar lo que va sucediendo en los procesos de enseñanza-aprendizaje que protagonizan (2011: 93).

Lo anterior puede verse afectado por diferentes factores que intervienen en la labor del profesor, y que desde la perspectiva de la OCDE están asociados a sobrecarga, diversificación y, por lo tanto, complejidad e intensificación de la tarea docente. Esta intensificación desde la perspectiva de Poggi, puede ser caracterizada por:

1) la obligación para los docentes de hacer más con menos recursos; 2) la disminución del tiempo que pasan con los alumnos; 3) la diversificación de sus papeles (docentes, psicólogos, policías, padres, motivadores, etc.), 4) la obligación del trabajo colectivo y de la participación en la vida de los establecimientos; 5) el manejo cada vez más pesado de públicos estudiantiles con dificultades; 6) finalmente exigencias cada vez más numerosas de las autoridades políticas y públicas para con los docentes, quienes deben comportarse como trabajadores industriales, es decir, fungir como mano de obra flexible, eficiente y de bajo costo (2013: 34).

Las prácticas pedagógicas corresponden a un elemento esencial para comprender la complejidad del proceso de enseñanza y de aprendizaje, ya que, a través de estas el docente ejerce su labor, por tanto, retroalimentarlas contribuye al fortalecimiento del ejercicio docente.

La retroalimentación busca generar cambios en los procesos de enseñanza y aprendizaje. Es necesario que esta se brinde de manera efectiva y en el momento oportuno, para lograr la motivación necesaria y así conseguir mejorar el desempeño y desarrollar experiencias de enseñanza más significativas. Este como se ha mencionado en ocasiones anteriores, es el fin último del proceso evaluativo, donde la retroalimentación cumple un rol fundamental, como afirma Tejedor, "la información proporcionada pone en marcha un mecanismo de retroalimentación de manera que se produce en el profesor una cierta disonancia o insatisfacción que le induce a cambiar" (2012:319).

La función pedagógica, formativa y reguladora que se espera de la evaluación, debe hacer frente a una práctica evaluativa que ha enfatizado el control punitivo de premios y castigos; con énfasis en el resultado y como mecanismo social de selección reforzando con ello el desarrollo de prácticas pedagógicas tradicionales que resultan interesantes de indagar a la luz de los requerimientos que plantea el decreto $\mathrm{N}^{\circ} 67 / 2018$, el que busca promover una visión de la evaluación, en contextos pedagógicos, como un aspecto intrínseco a la enseñanza, cuyo sentido fundamental es propiciar y apoyar los aprendizajes de los estudiantes. Desde esta perspectiva, la evaluación cumple un rol crucial en el monitoreo y acompañamiento del aprendizaje de los estudiantes y en la reflexión docente para la toma de decisiones pertinentes y oportunas respecto a la enseñanza. 
Con estos elementos teóricos en discusión, se llevó a cabo la investigación cuya novedad es aproximarse a la puesta en marcha del Decreto 67/2018, profundizando en la implementación de la evaluación formativa que desarrolla un grupo de profesores de educación básica en el contexto de la asignatura de Historia, Geografía y Ciencias Sociales, procurando fijar la posición que tienen sobre la evaluación como regulación continua del aprendizaje.

Por lo tanto, la investigación busca indagar la percepción respecto al concepto y finalidad de la evaluación formativa; el cómo se desarrolla en el contexto de la asignatura, precisando en la caracterización de los instrumentos; y en la determinación de los factores que condicionan su implementación.

\section{METODOLOGÍA}

La investigación se enmarca en un paradigma interpretativo, el cual tiene como finalidad para Latorre, Del Rincón y Arnal "comprender e interpretar la realidad, los significados de las personas, percepciones, intenciones, acciones" (2003: 44). Por tanto, este estudio busca comprender la realidad de los docentes, a través de un análisis, con respecto a cómo se desarrolla la evaluación formativa en la enseñanza de Historia, Geografía y Ciencias Sociales.

A su vez, esta, asume un enfoque cualitativo, de alcance descriptivo. Desde la perspectiva de Báez y Pérez, busca comprender los fenómenos que rodean a los participantes, profundizar en sus experiencias, miradas, opiniones y significados (2009), logrando descubrir cómo ellos en este caso perciben y desarrollan la evaluación formativa.

El diseño de esta investigación se sustenta en el estudio de casos múltiples, de carácter interpretativo, los cuales se caracterizan por reunir "tanta información sobre el problema como sea posible, con la pretensión de interpretar o teorizar acerca de un fenómeno" (Pérez 2008: 97).

Para la recogida de información se utilizó la entrevista individual semiestructurada, que adquiere la forma de un diálogo flexible (Flik 2007). Se dispuso el uso de esta aproximación metodológica, con guión de preguntas previamente validado, la que permitió acceder a datos descriptivos y a un abordaje interpretativo de los sujetos investigados.

Otro instrumento de recogida de información utilizado corresponde a la observación de clase. Para ello se observó tres clases impartidas por los docentes previamente entrevistados, en periodos de noventa minutos cada una, lo que corresponde a dos horas pedagógicas en la asignatura de Historia, Geografía y Ciencias Sociales.

Participaron en este estudio seis escuelas básicas representadas en sus profesores encargados de la asignatura de Historia, Geografía y Ciencias Sociales para el segundo ciclo básico. Ellos de manera voluntaria decidieron responder a catorce interrogantes planteadas a través de una entrevista semiestructurada de carácter individual, con el previo conocimiento y aceptación del consentimiento informado. Y, además, otorgaron la autorización para el desarrollo de la observación de clase, por medio de una pauta con diversos indicadores. Los entrevistados son en su totalidad profesores de enseñanza general básica.

Se utilizó la técnica de análisis de contenido (Ruiz 2009), a partir de la transcripción de entrevistas y el levantamiento de categorías acompañado de la evidencia empírica correspondiente para su posterior discusión teórica y conclusiones.

\section{RESULTADOS}




\section{Entrevistas:}

1. Concepto y finalidad de la evaluación formativa

Tabla 1. Concepto y finalidad de evaluación formativa

Pregunta
¿Qué entiende
usted por
evaluación del
aprendizaje?

¿Cómo entiende
usted el concepto
de evaluación
formativa?

¿Cuál cree usted es la finalidad de la evaluación formativa en la enseñanza de Historia, Geografía $\quad$ y Ciencias Sociales?

$\begin{aligned} & \text { Categorías } \\ & \text { - } \text { Proceso } \\ & \text { - } \text { Formativa } \\ & \text { - } \text { Permanente } \\ & \text { - } \text { Diagnóstico }\end{aligned}$

- Proceso continuo y gradual

- De progreso e interactiva Después de un contenido o unidad

- Mejorar proceso y resultado

- Retroalimentar y reorientar

- Formar personas ciudadanas en todas sus dimensiones

\section{Relatos}

"La evaluación del aprendizaje es un proceso formativo, permanente $\mathrm{y}$ continuo, que permite conocer los avances e identificar las limitaciones en el aprendizaje" (Sujeto,5)

"Proceso más continuo, más gradual, algo cotidiano, que uno hace siempre" (Sujeto, 2)

"Consiste en evaluar el progreso de los conocimientos del alumno de forma frecuente e interactiva. En las cuales los profesores pueden ajustar sus programas para satisfacer mejor sus necesidades educativas" (Sujeto, 5)

"Construir personas en todas las dimensiones y personas ciudadanas que se den cuenta, de la parte valórica, ética, moral de lo que es vivir en este mundo. Más allá de enseñarle a un niño de cómo ocurrieron los hechos, fechas, personajes, es más para no cometer los mismos errores en la historia" (Sujeto, 4)

"Reforzar y retroalimentar al estudiante y a partir de esto poder orientar también el proceso de enseñanza y aprendizaje hacia mejorar de alguna forma los aprendizajes del niño. Es decir, generar nuevas estrategias de enseñanza y aprendizaje para el estudiante para que logre cada vez avanzar más, esa es como la principal finalidad" ( Sujeto, 2)

(Fuente. Elaboración propia, 2019)

En el proceso de enseñanza y aprendizaje, la evaluación es un componente intrínseco, que se entiende como un proceso, es decir, evaluar no es un evento sino un conjunto de acciones permanentes en el aula. Cano sostiene que la evaluación hace referencia a "un proceso que utiliza diversidad de instrumentos e implica a diferentes agentes, con el propósito de proporcionar información sobre la progresión en el desarrollo de la competencia y sugerir caminos de mejora" (2008: 10).

El grupo de profesores estudiados, evidencia tener conocimiento respecto al concepto de evaluación del aprendizaje del mismo modo, aluden a que la noción de evaluación formativa, está vinculada al progreso que se ha de esperar en el proceso de enseñanza y aprendizaje de sus estudiantes. Por consiguiente, se puede dejar en claro que los entrevistados muestran percepciones complementarias respecto al concepto de evaluación 
formativa. Sin embargo, no logran tener claridad total respecto a los alcances de la evaluación formativa en relación a la necesaria regulación continua del aprendizaje. Desde el punto de vista teórico, Brookhar (2009) plantea que la evaluación formativa constituye el "proceso mediante el cual se recaba información sobre el proceso de enseñanza aprendizaje, que los maestros pueden usar para tomar decisiones instruccionales y los alumnos para mejorar su propio desempeño, y que es una fuente de motivación para los alumnos" (citado por Martínez, 2012, p. 4).

Tabla 2. Evaluación formativa en la enseñanza de Historia, Geografía y Ciencias Sociales

\begin{tabular}{|c|c|c|}
\hline Pregunta & Categorías & Relatos \\
\hline $\begin{array}{lrr}\text { En la asignatura de } & \text { de } \\
\text { Historia, Geografía } & \text { y } \\
\text { Ciencias Sociales, ¿Cómo } \\
\text { desarrolla usted la } \\
\text { evaluación formativa con } \\
\text { sus estudiantes? ¿Por qué? }\end{array}$ & $\begin{array}{ll}\text { - } & \text { Observación } \\
& \text { directa } \\
\text { - } & \text { Trabajo en clase } \\
\text { - } & \text { Producción de } \\
& \text { los estudiantes }\end{array}$ & $\begin{array}{l}\text { "Utilizo diferentes tipos de } \\
\text { evaluaciones, ya sea con } \\
\text { materiales concretos, material } \\
\text { audiovisual, esto según sus } \\
\text { estilos de aprendizaje, por lo } \\
\text { general para estas evaluaciones } \\
\text { usó como instrumento una pauta } \\
\text { de observación" (Sujeto, 5) }\end{array}$ \\
\hline $\begin{array}{lcc}\text { ¿De qué manera } & \text { la } \\
\text { evaluación formativa } & \text { le } \\
\text { permite monitorear } & \text { el } \\
\text { aprendizaje de } & \text { sus } \\
\text { estudiantes? ¿Por qué? } & \end{array}$ & \begin{tabular}{ll} 
- & \multicolumn{2}{l}{ Atender ritmos y } \\
& estilos de \\
aprendizaje \\
- & Diálogo \\
& pedagógico
\end{tabular} & $\begin{array}{l}\text { "Debiera monitorear el proceso } \\
\text { de cada estudiante, dependiendo } \\
\text { del ritmo y estilo de aprendizaje } \\
\text { de cada estudiante." (Sujeto, 4). } \\
\text { "Observó el progreso de sus } \\
\text { aprendizajes ya sea por } \\
\text { evidencia de trabajos escritos o } \\
\text { por medio de la observación en } \\
\text { los momentos de diálogos } \\
\text { pedagógicos" (Sujeto, 5) }\end{array}$ \\
\hline
\end{tabular}

(Fuente. Elaboración propia, 2019)

En la asignatura de Historia, Geografía y Ciencias Sociales que desarrollan estos profesores, la evaluación formativa se despliega a través de diversos instrumentos, que oscilan entre un rol pasivo del estudiante, donde se pone mayor énfasis en la participación del docente, y un rol activo, el cual ofrece mayor oportunidades y presencia a las percepciones, intereses y motivaciones del alumno.

El docente en esta asignatura declara monitorear el aprendizaje de sus alumnos, principalmente a través de la observación directa, ya que, esta herramienta le permite a la vez reflexionar sobre su práctica y reorientar la enseñanza de manera inmediata, para que todos los estudiantes logren cumplir con las metas de aprendizaje establecidas. Además, mediante el diálogo pedagógico que se establece en el aula, el profesor puede detectar impresiones y comportamientos de sus estudiantes. Y, en definitiva, conocer cómo sus alumnos van adquiriendo el aprendizaje.

Es importante destacar que la muestra en estudio hace hincapié a lo fundamental de evaluar el proceso del estudiante. Por consiguiente, utilizar una variedad de instrumentos permite atender a la diversidad que existe en el aula, considerando que cada estudiante aprende a través de diversos estilos y con diferentes ritmos de avanza y, por lo tanto, debe 
ser evaluado de maneras variadas. En efecto y coherente con lo que plantean los requerimientos del ministerio de Educación, "la educación debe ser adecuada a las distintas necesidades que van teniendo las y los estudiantes, y que, por tanto, debe diversificarse para asegurar la pertinencia de las experiencias formativas de los y las estudiantes. En consecuencia, la evaluación también debe adecuarse, ofreciendo oportunidades de desarrollo a todos y todas los y las estudiantes" (2018: 12).

Tabla 3. Desafíos que representa la evaluación formativa

\begin{tabular}{|c|c|c|}
\hline Pregunta & Categorías & Relatos \\
\hline $\begin{array}{l}\text { ¿Cuáles son los desafíos que } \\
\text { enfrenta al incorporar la } \\
\text { evaluación formativa a la } \\
\text { práctica pedagógica? }\end{array}$ & $\begin{array}{ll}\text { - } & \text { Proporcionar } \\
& \text { evidencia constante } \\
\text { - } & \text { Generar nuevas } \\
& \text { estrategias } \\
\text { - } & \text { Instruirnos para una } \\
& \text { visión común }\end{array}$ & $\begin{array}{l}\text { "Ser capaces de atender } \\
\text { con este tipo de evaluación } \\
\text { a la totalidad de } \\
\text { estudiantes, dar evidencia } \\
\text { constante del proceso y } \\
\text { progreso a UTP y a las } \\
\text { familias, me pregunto } \\
\text { ¿seré capaz?" (Sujeto, 5) } \\
\text { "Que cada día hay que } \\
\text { generar nuevas estrategias } \\
\text { de enseñanza para la } \\
\text { diversidad de estudiantes } \\
\text { que tenemos" (Sujeto, 2) }\end{array}$ \\
\hline
\end{tabular}

(Fuente. Elaboración propia, 2019)

Al momento de desarrollar la evaluación formativa en el aula, los profesores entrevistados reconocen la existencia de diversos factores que intervienen en su implementación, dentro de los cuales están los que favorecen y los que limitan su ejecución. Con respecto a los primeros, se destaca el buen clima que se genera en el aula para propiciar el aprendizaje de los estudiantes, atendiendo así la diversidad que se pueda presentar. Ello se corresponde con el planteamiento teórico de López quien afirma que "dentro de un espacio de convivencia democrática, el profesor debe tratar de crear un clima que facilite la participación, el espíritu crítico, la honestidad, la relación, el respeto, la responsabilidad, etc." (2009:).

De igual forma, surgen factores limitantes para la implementación de esta, que en su totalidad corresponden a factores externos tales como: disciplina, que se vería afectada por casos de conducta; tiempo disponible, justificando su carga laboral y el uso de la valuación con fines de calificación y castigo, asociando ellos a la idea de que evaluar es generar condicionamiento en sus estudiantes. Esto último, puede vincularse desde el punto de vista teórico con el planteamiento de Martínez quien señala que "la dificultad para poner en práctica en las aulas los principios de la evaluación formativa parece deberse, pues, a la influencia sobre las prácticas de factores de diferente peso y antigüedad, que individualmente podrían considerarse menores" (2013, p.5).

Al referirse a los desafíos que enfrentan los docentes al incorporar la evaluación formativa en sus prácticas pedagógicas, los sujetos advierten que estos desafíos se asocian a que la naturaleza de la evaluación formativa exige disponer y propiciar evidencias constantes en cuanto al aprendizaje de los estudiantes, con los costos que ello implica y los beneficios 
que para el estudiantado tendría. En efecto, la enseñanza tendrá la posibilidad cierta de atender de manera efectiva a la diversidad que hay dentro de un aula. Cabe señalar que:

El principal desafío que enfrentamos actualmente en las escuelas en lo relativo a evaluación es asegurar que las prácticas sanas lleguen a cada aula, que las evaluaciones se usen para beneficiar a los alumnos... Este reto ha permanecido sin ser atendido por décadas y parece que ha llegado el tiempo de conquistar esa última frontera de la evaluación: el uso efectivo de evaluación formativa para apoyar el aprendizaje (Stiggins 2007, citado por Martínez 2013:129).

Tabla 4. Instrumentos de evaluación formativa

\author{
Pregunta \\ ¿Qué instrumento y/o técnica \\ de evaluación considera usted \\ más efectivo al momento de \\ evaluar el progreso de los \\ aprendizajes de sus \\ estudiantes? ¿Por qué?
}

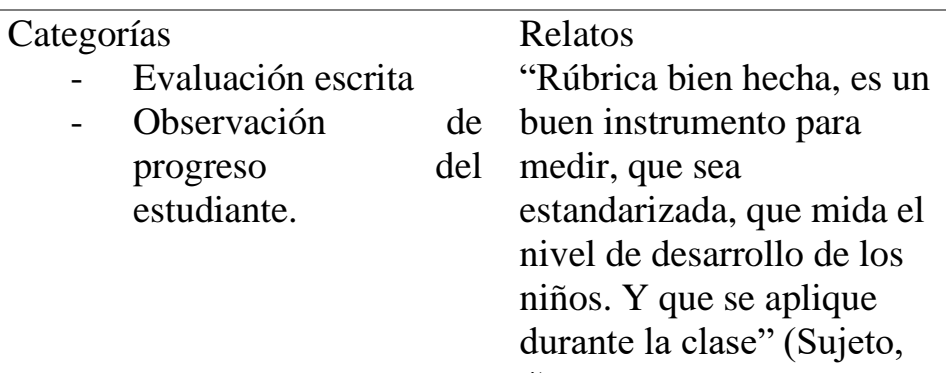

\author{
Relatos \\ "Rúbrica bien hecha, es un \\ estandarizada, que mida el \\ nivel de desarrollo de los \\ niños. Y que se aplique \\ durante la clase" (Sujeto,
}

de buen instrumento para

del medir, que sea

(Fuente. Elaboración propia, 2019)

Al seleccionar instrumentos para evaluar formativamente en el aula, los docentes entrevistados consideran pertinente valorar los estilos de aprendizaje del estudiante, para decidir y seleccionar una herramienta evaluadora acorde a sus capacidades y necesidades. Del mismo modo, debe tener en cuenta el contexto del aula, y el tiempo que dispone el profesor para lograr así trabajar con la realidad de cada curso. Es por esto que se debe tener en cuenta lo que señala Timón "entendemos la diversidad del alumnado en un sentido amplio. Se trata de un elemento enriquecedor y, desde luego, es una de las circunstancias más relevantes que es preciso tener en cuenta desde un punto de vista educativo al planificar y desarrollar los procesos de enseñanza/aprendizaje" (2010: 9).

Por consiguiente, declaran que no hay claridad de qué instrumento sea el más efectivo para evaluar el progreso del aprendizaje. Pero cabe destacar que la evaluación formativa constituye una poderosa herramienta educativa, en la cual se distinguen dos tipos:

evaluación formativa formal, que incluye las preguntas o actividades planeadas por el docente como parte de una lección o unidad de trabajo, con el objetivo de obtener evidencia de aprendizaje en un grupo de alumnos; y evaluación formativa informal, la cual surge de manera espontánea en el aula y tiende a enfocarse en la obtención de información sobre el aprendizaje cuando sea que la oportunidad se presente (Cowie y Bell 1999, citado por Talanquer 2015: 178).

Observación de Clases:

Tabla 5. Análisis observación de clases: La evaluación formativa en la clase de Historia, Geografía y Ciencias Sociales 


\begin{tabular}{|c|c|c|c|}
\hline \multirow[t]{2}{*}{ Indicadores } & \multicolumn{3}{|c|}{ Escala } \\
\hline & 1 & 2 & 3 \\
\hline Clarifica objetivo de aprendizaje en conjunto con estudiantes. & 2 & 5 & 11 \\
\hline $\begin{array}{l}\text { Vincula el tema anterior con el nuevo (preguntas, imágenes, videos) } \\
\text { captando la atención e interés por parte de sus alumnos. }\end{array}$ & 4 & 5 & 9 \\
\hline $\begin{array}{l}\text { Formula preguntas que permiten al estudiante el proceso de } \\
\text { comprensión y ampliación de su aprendizaje }\end{array}$ & & 6 & 12 \\
\hline $\begin{array}{l}\text { Responde a las interrogantes e inquietudes de sus estudiantes } \\
\text { favoreciendo la comprensión y ampliación de su aprendizaje }\end{array}$ & 1 & 8 & 9 \\
\hline Monitorea el desarrollo de las actividades en el transcurso de la clase & 4 & 4 & 10 \\
\hline $\begin{array}{l}\text { Reestructura la clase a partir de los aciertos y errores de sus } \\
\text { estudiantes }\end{array}$ & 9 & 5 & 4 \\
\hline $\begin{array}{l}\text { Realiza actividades de cierre, apropiados (sistematización, síntesis, } \\
\text { cognición y metacognición), abriendo nuevos desafíos o tareas a } \\
\text { realizar. }\end{array}$ & 5 & 10 & 3 \\
\hline $\begin{array}{l}\text { Refuerza los logros y avances de los alumnos, en forma verbal y/o } \\
\text { escrita en el libro de clases (estímulos, felicitaciones, anotaciones } \\
\text { positivas) }\end{array}$ & 1 & 4 & 13 \\
\hline
\end{tabular}

(Fuente. Elaboración propia, 2019)

\begin{tabular}{|c|l|}
\hline \multicolumn{2}{|l|}{ Escala de Apreciación } \\
\hline 3 & Siempre (Cumple correctamente con el indicador establecido) \\
\hline 2 & Casi Siempre (No existe un dominio amplio y profundo del indicador). \\
\hline 1 & $\begin{array}{l}\text { Rara Vez (No cumple adecuadamente presentando falencias en el } \\
\text { indicador). }\end{array}$ \\
\hline
\end{tabular}

En una concepción de enseñanza en que aprender es un proceso complejo a través del cual el alumno va construyendo y reestructurando su conocimiento a partir de las actividades que lleva a cabo, es clave el papel que juega la evaluación. En ese sentido, la observación realizada en aula pone de manifiesto la presencia de una evaluación formativa por parte de los profesores, sustentada en las siguientes acciones de aula:

- Clarifica objetivo de aprendizaje en conjunto con estudiantes, intención de favorecer la comprensión de lo que se pretende aprender en el desarrollo de la clase.

- Formula preguntas que permiten al estudiante el proceso de comprensión y ampliación de su aprendizaje, como mecanismo que permite ir verificando la compresión que los estudiantes logran del contenido y del aprendizaje comprometido.

- Monitorea el desarrollo de las actividades en el transcurso de la clase, para ayudar en la apropiación de los aprendizajes, resolviendo inquietudes y alentando la participación en el desarrollo de las actividades de la clase. 
- Refuerza los logros y avances de los alumnos, en forma verbal y/o escrita en el libro de clases (estímulos, felicitaciones, anotaciones positivas), como dispositivo para motivar el compromiso de los estudiantes con su aprendizaje.

Estas prácticas desarrolladas en el aula, muestran el reconocimiento por parte de los profesores, de los cambios que han de ir incorporando en el proceso de enseñanza y aprendizaje para asegurar que todos los alumnos aprendan de manera apropiada. Son acciones insertas en el proceso, ya sea en su inicio, durante él o al final, con la finalidad de mejorar el aprendizaje.

\section{CONCLUSIONES}

La evaluación es un componente fundamental del proceso de enseñanza y aprendizaje, condiciona qué, cuándo y cómo se enseña, más aún cuando hoy enfrentando importantes desafíos asociados a diversidad presente en el aula.

La evaluación orientada hacia un sentido pedagógico es uno de los principales desafíos que el decreto $\mathrm{N}^{\circ} 67 / 2018$ pretende implementar de manera sistemática y generalizada desde marzo del 2020. El interés investigativo se sustenta en el hecho que la evaluación formativa es un concepto que fue introducido el año 1967 por M. Scriven para dar cuenta de los procedimientos utilizados por los profesores con la intención de adaptar el proceso didáctico a los progresos, dificultades y requerimientos de aprendizaje observados en sus alumnos. En ese sentido, es un concepto y, por tanto, esperemos también una práctica de larga data en el sistema educativo, que hoy se intenta relevar; razón por la cual la investigación pretende explorar el estado de situación al respecto.

Es posible concluir que la conceptualización de la evaluación ya sea formativa y/o del aprendizaje, está referida a la noción de proceso, que tiene las características de continua y gradual. La evaluación requiere de una secuencia de acciones que se deben realizar de manera permanente para determinar el desempeño de los estudiantes: partiendo desde el diagnóstico al que se le puede dar un énfasis formativo, retroalimentando a los estudiantes sobre ciertas debilidades que se pueden encontrar. Además, de tomar conciencia de la manera en que los estudiantes aprenden, y así de este modo responder a sus estilos de aprendizaje.

Desde el punto de vista conceptual, las entrevistas ponen de manifiesto la existencia de una clara diferencia entre los términos de medición y evaluación, ya que, la primera apunta directamente a cuantificar el aprendizaje esperado a través de una calificación, mientras que la segunda, hace referencia a constatar el progreso continuo y permanente del aprendizaje de los estudiantes, es decir, evaluar formativamente. Junto a ello, también aluden a que su finalidad es orientar y reestructurar el proceso educativo de cada estudiante, utilizando las diversas herramientas y estrategias para que de esta manera cada niño pueda lograr su proceso de aprendizaje, y con ello, contribuir al mejoramiento del quehacer docente.

Las entrevistas y principalmente las observaciones de clases permiten sostener que el profesorado implementa la evaluación formativa en la mayoría de sus clases, declarando que ejecutan un constante monitoreo sobre el aprendizaje del alumnado, a través de la observación directa y la formulación de preguntas. Además, reconocen que esta se desarrolla en todo momento, ya sea al inicio de la clase, en la activación de conocimientos previos, en el desarrollo, cuando se realizan diversas actividades o en el cierre, cuando el profesor sintetiza y retroalimenta. Sumado a esto, también se demostró que estos profesores, desarrollan la evaluación formativa al momento de realizar diversos trabajos 
en clases, que involucren producciones de los estudiantes, como maquetas, exposiciones, guías, entre otros, que en definitiva aportan insumos respecto a los logros y dificultades que van experimentando los estudiantes, con la intención de introducir los ajustes y regulaciones que se estimen convenientes.

Nos llama la atención, y resulta alentador reconocer que el profesorado investigado es consciente de la diversidad que existe en el aula en términos de distintos estilos de aprendizaje que se pueden presentar y que, por tanto, la enseñanza debe estar orientada a atender la heterogeneidad. Y como la evaluación es un componente fundamental que sustenta al proceso educativo, esta también debe ir dirigida a utilizar diversos instrumentos para recoger información, se pretende que el estudiante tenga una participación activa en su proceso de aprendizaje, con el objetivo de otorgar autonomía a través de una autoevaluación y coevaluación.

La retroalimentación cumple un rol fundamental, puesto que a través de esta el docente permite al estudiante saber qué hacer para mejorar y disminuir la zona de desarrollo próximo. Se evidenció que los profesores de este estudio, realizaban retroalimentación constante, para localizar dificultades y de esta manera reorientar la modalidad de enseñanza.

Como factores claves para el desarrollo de evaluación formativa, se encuentran como más destacados, el buen clima dentro del aula y el apoyo por parte de la familia. En cambio, respecto a los factores que limitan, estos se encuentran representados por el tiempo disponible en relación a las tareas que implica el desarrollo de la evaluación formativa, vale decir, debido a la escasez que se posee para planificar, crear y además aplicar efectivamente una evaluación formativa. Desde ese punto de vista, se podría pensar, la existencia en el sistema escolar de una especie de paradoja con respecto a evaluación, ya que, por una parte, se declara la necesidad de hacer evaluación formativa, y por tanto atender efectivamente a las diferencias individuales, sin embargo, por otra parte, se sigue dando énfasis a evaluar para los resultados, lo que termina por generar más de una confusión en el profesorado.

No obstante, al contrastar los resultados de la entrevista, con lo evidenciado en las observaciones de clases, donde se observan actuaciones pedagógicas alineadas con la evaluación formativa, los factores limitantes de la evaluación formativa parecen ser de otra naturaleza, tales como: disposiciones, creencias y una constante reflexión de su labor, para asumir cuotas de responsabilidad en lo que a implementación de evaluación formativa se refiere, ya que, se infiere que el profesor piensa en que las dificultades siempre son de carácter externo.

Se infiere que los instrumentos que los docentes utilizan están representados fundamentalmente por técnicas de observación y de gran carga reflexiva, por parte de los profesores. Otra de las características que presentan los instrumentos utilizados, es que permiten al docente potenciar la habilidad de reestructurar oportunamente la clase a partir del monitoreo y de las evidencias recogidas en el momento, como por ejemplo las preguntas dirigidas.

Considerando que, el uso formativo de la evaluación busca una regulación continua del aprendizaje y que además pretenden demostrar que aprender es un proceso complejo, a partir del cual el sujeto podría construir su conocimiento de manera individual, los profesores administran instrumentos de evaluación variados en el aula, los cuales no quedan circunscritos a las pruebas.

Se logra establecer que, en la práctica pedagógica, es posible observar el uso de la evaluación formativa, particularmente a nivel de instrumentos y uso de la evaluación con 
fines de retroalimentación; sin embargo, se evidencia la necesidad de fortalecer conceptualmente esta temática entre el profesorado, particularmente en lo que a función pedagógica de la evaluación se refiere. Es valorable, el esfuerzo que realiza el profesorado por atender a la diversidad presente en el aula, desplegando una evaluación que le permite ir recogiendo información que orienta la toma de decisiones.

En consecuencia, se concluye el potencial pedagógico que presenta la evaluación, un buen uso puede constituir una herramienta llamada a formar, es decir, posibilitar que todos y todas aprendan de manera significativa respetando ritmos y estilos de aprendizaje. Sin embargo, mal utilizado, puede reducirse a un arma que deforma, discrimina y no potencia el aprendizaje, lo que ocurre cuando esta es utilizada como un mecanismo de amenaza o cuando la evaluación sanciona el error. El uso que se le dé a la evaluación dependerá del objetivo que se quiera alcanzar y fundamentalmente, a los conocimientos y creencias del profesorado.

Así mismo, se considera que uno de los factores que pueden influir en la implementación de la evaluación formativa, se relaciona con la formación inicial de cada docente, ya que, si no se genera un conocimiento previo, probablemente dificultará un eficaz desarrollo en la práctica de evaluación formativa. Esto debería estar sustentado en la entrega de herramientas necesarias para promover el desarrollo profesional continuo, con un enfoque centrado en el aprendizaje y evaluación, que favorezcan el progreso de los estudiantes.

Finalmente, se hace necesario enriquecer las competencias de los profesores para que puedan desarrollar la evaluación formativa, asegurando un cambio efectivo en la práctica de la evaluación, fortaleciendo así la regulación continua del aprendizaje.

\section{REFERENCIAS}

Agencia de Calidad de la Educación. (2018, febrero). Nuevo Sistema Nacional de Evaluación de Aprendizajes. Recuperado 4 noviembre, 2019, de http://archivos.agenciaeducacion.cl/Sistema_Nacional_de_Evaluacion_17abr.pd

Alliaud, A (2011). "Narración de la experiencia: práctica y formación docente" - Revista Reflexão e Ação 19, 92-108.

Báez y Pérez, J (2009): Investigación cualitativa (2 ${ }^{\mathrm{a}}$ ed.) - España: ESIC Editorial.

Calatayud, M. (2019). "Orquestar la Evaluación Inclusiva en los Centros Educativos. ¿Por dónde Empezar?”. Revista Internacional de Educación para la Justicia Social, 8(2), 165-176.

Cano, M. (2008). "La evaluación por competencias en la educación superior". Revista de currículum y formación del profesorado, 12, 1-16.

Chile, Ministerio de Educación (2018). Política para el fortalecimiento de la evaluación en el aula. Santiago, Chile.

Chile, Ministerio de Educación. (2019). Orientaciones para la elaboración del reglamento de evaluación. Santiago, Chile: Unidad de currículum y evaluación

Chile, Ministerio de Educación. (s.f.). Curriculum Nacional. Recuperado el Octubre de 2019, de https://www.curriculumnacional.cl/614/w3-article-14606.html

Chile, Ministerio de educación (s.f.). Orientaciones para la implementación del decreto 67/2018 de evaluación, calificación y promoción escolar. Santiago, Chile: Autor, Unidad de currículum y evaluación 
Contreras, G. (2017): "Formación de profesores en evaluación del aprendizaje" en De los fundamentos a las prácticas: algunos desafíos en la formación inicial docente Garrido, J. (119 - 132). Valparaíso: Ediciones Universitarias de Valparaíso.

Decreto $N^{\circ} 67$. Diario Oficial de la República de Chile, Santiago, Chile, 31 de diciembre de $2018 . \quad$ Recuperado de https://www.diariooficial.interior.gob.cl/publicaciones/2018/12/31/42242/01/1521939.p df

Flick, U. (2007): Introducción a la investigación cualitativa -España: Morata.

Latorre, A., Del Rincón, D., \& Arnal, J. (2003): Bases metodológicas de la investigación educativa - España: Experiencia.

López, V. (2009): Evaluación formativa y compartida en Educación Superior: Propuestas, técnicas, instrumentos y experiencias - España: NARCEA S.A DE EDICIONES.

Martínez, F. (2012). "La evaluación formativa del aprendizaje en el aula en la bibliografía en inglés y francés: Revisión de literatura" - Revista mexicana de investigación educativa 17(54), 849-875.

Martínez, F. (2013). "Dificultades para implementar la evaluación formativa: Revisión de literatura" - Perfiles educativos 35(139), 128-150.

Muñoz, J., Villagra, C., \& Sepúlveda, S. (2016). \#Proceso de reflexión docente para mejorar las prácticas de evaluación de aprendizaje en el contexto de la educación para jóvenes y adultos" - FOLIOS 44 (2), 77-91.

Pérez, G. (2008): Investigación cualitativa. Retos e interrogantes (6 $6^{\mathrm{a}}$ ed.) - España: La Muralla.

Poggi, M. (2013): Políticas docentes: formación, trabajo y desarrollo profesional Argentina: Publicaciones, IIPE-UNESCO.

Talanquer, V. (2015). "La importancia de la evaluación formativa" - Educación química 26(3), 177-179.

Tejedor, F. (2012). "Evaluación del desempeño docente" - Revista Iberoamericana de Evaluación Educativa 5(1), 318-327.

Timón, L. (2010): Atención a la diversidad en el marco escolar: Propuestas de integración para alumnos con necesidades de apoyo educativo - España: Wanceulen.

Zabalza, M. (2007): Competencias docentes del profesorado universitario ( $2^{\mathrm{a}}$ ed.). España: Narcea 